Elsevier

HRR 00802

\title{
Trimethyltin ototoxicity: evidence for a cochlear site of injury
}

\author{
Laurence D. Fechter*, John S. Young and Alfred L. Nuttall \\ Neurotoxicology Program, Department of Environmental Health Sciences, Johns Hopkins University School of Hygiene, \\ Battimore, MD 21205, and Kresge Hearing Research Institute, University of Michigan Medical School, Ann Arlor, MI 48109. U.S.A.
}

(Received 3 October 1985; accepted 2 March 1986)

\begin{abstract}
The environmental contaminant, trimethyltin (TMT), produces a profound elevation in tone intensity necessary to inhibit the acoustic startle reflex in laboratory animals which recovers over a prolonged period except at very high frequencies. The recovery that is observed does not begin until 3 to 5 weeks after a single acute administration depending upon dosage. As opposed to the very temporary threshold shifts by the salicylates and loop diuretics or the permanent and progressive ototoxicity resulting from aminoglycoside antibiotics the time course for recovery of acoustic startle reflex inhibition after TMT appears to be an anomaly for a chemical ototoxicant. In terms of the duration of loss only. this pattern appears similar to that sometimes observed after noise exposure. The current investigation replicates the finding that recovery of acoustic startle reflex inhibition after TMT is frequency related in that only the highest frequency impairment appears to be permanent. While this frequency dependence suggests a cochlear locus of injury, both the known neurotoxic effects of TMT and the time course of the behavioral impairment suggest a more central locus of injury. Compound action potential and cochleat microphonic recordings made from the round wndow in the current study confirm a preferential high frequency effect of TMT and demonstrate a significant cochlear component to the ototoxic affects of this agent.
\end{abstract}

ototoxicity, peripheral auditory damage, trimethyltin, reflex modification audiometry, compound action potential, cochlear microphonic

\section{Introduction}

A series of recent investigations have identified a variety of chemicals present in the environment as commercial products, chemical intermediaries, and contaminants which are potent ototoxic agents. These include organic solvents such as toluene (e.g., Rebert et al., 1983; Pryor et al., 1983) and hexane (e.g. Rebert et al., 1982, 1983) and a variety of inorganic and organic metals including the anti-neoplastic agent, cis-diaminedichloroplatinum (Fleischman et al., 1975) and the environmental contaminants, methyl mercury (Falk ct al., 1974; Wu et al., 1985), lead acetate (Yamamura et al., 1984). and trimethyltin (Young and Fechter, 1986). To the extent that specific injury is produced to auditory structures by such

\footnotetext{
* To whom correspondence should be addressed at the Johns Hopkins Universily.
}

agents, environmental toxicants may have substantial value in hearing research. In addition to public health and industrial medicine concerns raised by these newly recognized ototoxic agents, these compounds sometimes produce unique patterns of hearing loss which may be of use in understanding the mechanisms of cochlear damage and in predicting other compounds which may also be ototoxic.

We have recently reported (Young and Fechter, 1986) that trimethyltin chloride (TMT), produces a novel ototoxicity which is characterized by a very persistent, but partially reversible elevation in tone intensity necessary to inhibit the acoustic startle reflex. This impairment seems to preferentially disrupt sensitivity to high frequency tones (although our initial study employed tests only at 10 and $40 \mathrm{kHz}$ ) and to produce a loudness-recruitment-like phenomenon suggesting a cochlear location for the damage. These findings closely parallel results obtained using Neomycin in an identical 
behavioral test procedure (Young and Fechter, 1983). However, as opposed to various ototoxic drugs whose principal site of damage appears to be in the cochlea, recovery of normal function was first noted three weeks after administration of the toxicant and was essentially stable at five weeks after exposure. Residual loss was observed with higher TMT doses and at the highest frequency tested $(40 \mathrm{kHz})$. This time course for recovery is more akin to that sometimes observed following noise exposure than to the very transient or else permanent effects of chemical agents. The archetypical chemical ototoxicants, the aminoglycoside antibiotics, produce permanent hearing loss which may even progress after dosing has ceased. Salicylates, quinine, and loop diuretics typically produce transitory disturbances of hearing which last for several hours or days following dosing.

Data bearing upon the general ototoxicity of organic tins is limited at present. A preliminary report of triethyltin (TET as opposed to TMT) ototoxicity indicated disruption of compound action potentials and the cochlear microphonic recorded from the round window and histological evidence of profound stria vascularis injury (Evans et al., 1984). A full report of this work has not been published as yet and exhaustive efforts to replicate the findings using behavioral measures of hearing failed to uncover a significant effect of TET (Fechter and Young, 1983). Leake-Jones and Rebscher (1983) found that a tin containing catalyst used in curing the silastic carrier for a cochlear implant device employed in cats produced profound cochlear inflammation with damage likely to disrupt cochlear function. The compound was nominally identified as stannous octoate. A preliminary report of TMT exposure showing large disruptions in pure tone detection thresholds in rats has been published (Young and Fechter, 1986). The organotins are widely used in the manufacture of plastics and polyurethane foam and are commonly used as anti-fouling agents in marine paints (Wilkinson, 1984). While TMT is not itself of commercial interest in these instances, its presence as a precursor and contaminant has been identified. Thus, at present the ototoxicity of TMT is of interest as a heuristic for identifying general mechanisms of ototoxicity and as an exemplar of a group of structurally similar organometals with possible ototoxic effects.

The current study was designed to further de scribe the frequency specificity of behavioral loss and recovery produced by TMT using audiometric testing over the range of $2.5-40 \mathrm{kHz}$ just prior to and then at several time intervals following toxicant administration. In addition, measurement of the compound action potential (CAP) and cochlear microphonic (CM) was made in another group of subjects exposed to TMT in order to determine whether a cochlear site of injury could be adduced for the behavioral effects of TMT.

\section{Methods}

\section{Subjects}

The subjects were $300-350 \mathrm{~g}$ male Long Evans hooded rats. Subjects used for behavioral studies were housed in single cages with free access to food and water and under $12: 12 \mathrm{~h}$ diurnal lighting. Behavioral evaluations were performed on eight subjects, four of which were treated with 4 $\mathrm{mg} / \mathrm{kg}$ TMT. Subjects used for CAP and CM measurement were group housed. Electrophysiologic studies were conducted using seven subjects treated with $5 \mathrm{mg} / \mathrm{kg}$ TMT and seven saline controls. A dose of $5 \mathrm{mg} / \mathrm{kg}$ TMT was selected for the electrophysiological study because preliminary behavioral data suggested that the $4 \mathrm{mg} / \mathrm{kg}$ TMT dose might not produce significant loss.

\section{Behavioral testing}

The behavioral assessment of auditory thresholds was conducted using the reflex modification audiometry technique (Young and Fechter, 1983; Fechter and Young, 1983) in which the efficacy of pure tones in inhibiting a behavioral reflex is employed to assess detection thresholds for these pure tones. It is essential to point out that the pure tone stimuli used in establishing auditory thresholds are low intensity stimuli which modulate the startle reflex rather than high intensity auditory stimuli which would be necessary to elicit a startie reflex. Reflex modification results from the presentation of such low intensity stimuli just prior to reflex elicitation. The occurrence of preliminary stimulus inhibition cannot be explained on the basis of neuronal refractoriness, the middle 
ear reflex or classical conditioning. Both tactile and visual stimuli are equally effective in inhibiting an acoustic startle reflex and the time interval between the two stimuli would not favor learning. Reflex modification phenomena have been reported for a variety of mammalian and non-mammalian species using several different reflexes (c.f. Hoffman and Ison, 1980; Ison and Hoffman, 1982).

Subjects received a series of 880 trials each day consisting of baseline and test trials. The former are presentations of a $105 \mathrm{dBA}$ white noise burst of $20 \mathrm{~ms}$ duration designed to elicit a whole body startle reflex (160 trials). These are interspersed among test trials in which a $200 \mathrm{~ms}$ pure tone with $5 \mathrm{~ms}$ rise--decay times precedes the startle stimulus by $10 \mathrm{~ms}$ (offset to onset). Pure tone preliminary stimuli such as these have been shown to reduce the amplitude of the startle reflex elicited by the noise burst as a function of their intensity. Thus, the most intense preliminary stimuli, which are well above detection threshold for normal subjects, produce profound inhibition of the startle reflex elicited by a $105 \mathrm{dBA}$ white noise burst while less intense preliminary stimuli produce progressively less inhibition in the response to the same white noise burst.

For each test, individual subjects were placed into spring-mounted hardware cloth cages $(22 \times$ $10 \times 9.5 \mathrm{~cm}$ ) located within sound-isolated test chambers in an IAC double-walled audiometric room. Determination of reflex amplitude was performed by measuring the voltage induced in a wire coil by the movement of a magnet attached to the cage. The voltage was proportional to the speed of vertical movement imparted to the cage by the subject's response. The voltage was integrated over a $50 \mathrm{~ms}$ interval which began with the startle stimulus presentation.

Test trials consisted of randomly ordered presentations each of 9 tone frequencies $(2.5-40 \mathrm{kHz}$ in half-octave steps) presented at 19 intensities in $5 \mathrm{~dB}$ steps. On a given experimental day subjects received all 9 test frequencies. Each individual frequency-intensity combination was presented four times during a daily test session. Pure tone stimuli were delivered via a Realistic Model 401375 leaf-tweeter located $16 \mathrm{~cm}$ above the test cage. The $105 \mathrm{dBA}$ white noise stimuli were de- livered by two piezoelectric speakers located on either side of the cage. All stimulus intensity measurements were made free field using either a Brüel \& Kjaer $1 / 4$ or $1 / 2$ inch condenser microphone placed at the approximate level of the rats' ears.

Because of the large number of tone frequencies and intensities employed, it was necessary to test each subject daily for 14 days. These tests were conducted prior to TMT administration and then starting at $0,6,12$ and 18 weeks after exposure. The data from each 14 day test block were subjected to a square root transformation and a curve fitting procedure using a series of cubic polynomials. Such a function, referred to as a knotted spline function. provides a better fit of the data than a linear regression model. This statistical procedure was undertaken because the function relating reflex inhibition to pure tone intensity is somewhat sigmoidal rather than linear although the precise shape of the function varies across animals. The development of this model and the procedure for estimation of variance in fitting the curve have been more fully described elsewhere (Young and Fechter, 1983; Sheppard et al., under review). The detection threshold was defined as the point along the function yielding $15 \%$ reflex inhihition. In addition to providing a statistically reliable estimate of significant reflex inhibition, such a definition provides estimates of detection thresholds which are rather similar to those found using traditional operant behavioral techniques (e.g., Kelly and Masterton, 1977 for rats; Prosen et al., 1978 for guinea pigs). These threshold estimates are stable over repeated testing generally yielding apparent shifts of less than $5 \mathrm{~dB}$ (Young and Fechter. 1983). Further appropriate shifts specific to high frequency tones are found following aminoglycoside treatment (Young and Fechter, 1983). These findings argue for the validity of reflex modification methods as a behavioral measure of hearing.

\section{Electrophysiologic recordings}

Subjects received $5 \mathrm{mg} / \mathrm{kg}$ TMT or saline and then 7 days later were anesthetized by i.m. administration of ketamine $(87 \mathrm{mg} / \mathrm{kg})$ and xylazine $(13$ $\mathrm{mg} / \mathrm{kg}$ ). Subjects were tracheotomized, but did not require artificial respiration. Body tempera- 
ture was maintained at $37 \pm 1^{\circ} \mathrm{C}$ by a dc heating pad controlled by a rectal probe. The auditory bulla was then opened using a ventral approach. A 40 gauge $\mathrm{Ag}$ wire electrode insulated to about 2 $\mathrm{mm}$ from the tip was placed on the round window with a $\mathrm{Ag}-\mathrm{AgCl}$ reference placed in the neck muscle. The stimuli were pure tones of $1-40 \mathrm{kHz}$ presented by means of a $1 / 2$ inch Brüel $\&$ Kjaer microphone located within a sound speculum fitted into the external auditory meatus after removal of the pinna. Tone intensities were calibrated using a $1 / 2$ inch recording microphone attached to a probe tube within the speculum. The intensity of $10 \mathrm{~ms}$ tone pips ( $1 \mathrm{~ms}$ rise-decay time) was varied until a just observable $\mathrm{N}_{1}$ (approx. $10 \mu \mathrm{V}$ ) response was observed. The CM threshold was defined as a pure tone intensity generating a CM response of $1 \mu \mathrm{V}$ RMS. Comparisons of thresholds were made between groups using an analysis of variance.

\section{Results}

Table I presents the initial pure tone thresholds determined behaviorally prior to TMT and saline administration. Group assignments were made so as to produce roughly equivalent hearing thresholds in both groups and it is evident that systematic differences did not exist at this point in the experiment. The pure tone intensities necessary to achieve reflex modification in this study are quite comparable both in terms of sensitivity and shape to those obtained using traditional behavioral methods in rats (Kelly and Masterton, 1977).

The behavioral tests with TMT produced clear auditory threshold shifts in subjects from their pre-exposure pure tone thresholds (see Fig. 1). The shifts seen in the TMT subjects at the initial post-exposure test appear to occur rather uniformly across all frequencies although they are marginally greater at the higher frequencies tested.

Fig. 1 also shows the threshold shift observed at the second post-exposure tests conducted at $6-8$ weeks after TMT. It is apparent that the TMT-induced threshold shift was reversed at all but the highest test frequency used. No further recovery was observed at additional tests $12-14$ or 18-20 weeks following exposure (data not presented). For comparison and to study further the stability of sensory thresholds produced using reflex modification audiometry, the apparent shift in pure tone thresholds is presented for saline control subjects at the same time intervals as used for TMT-exposed rats (see Fig. 2). The most extreme average 'threshold shift' for controls was of $3 \pm 5 \mathrm{~dB}$ SPL in the first post test and $7 \pm 9 \mathrm{~dB}$ SPL on the second post test. In both cases, these shifts occurred at the lowest test frequency $(2.5$ $\mathrm{kHz}$ ). An analysis of variance confirmed significant differences between groups ( $F=13.50$. $P<0.05)$ and a significant test session by group interaction $(F=8.26, P<0.05)$ which reflects the stability of thresholds in control subjects contrasted with the threshold shifts observed in the TMT-treated group.

Trimethyltin also produced variable, but generally large depressing effects upon the vigor of startle responses elicited on trials not containing a preliminary stimulus. Baseline startle reflex values were depressed from the second to fourth week after TMT between -22 and $-91 \%$ in individual rats and ranged from -14 to $+29 \%$ in control

TABLE I

MEAN DETECTION THRESHOLD ( $\tilde{x})$ IN dB SPL AND STANDARD ERROR OF THE MEAN (S.E.) IN dB FOR PURE TONES DETERMINED BY REFLEX MODIFICATION AUDIOMETRY IN RATS PRIOR TO ADMINISTRATION OF TMT OR SALINE

\begin{tabular}{|c|c|c|c|c|c|c|c|c|c|c|}
\hline & & \multicolumn{9}{|c|}{ Frequency $(\mathrm{kHz})$} \\
\hline & & 2.5 & 3.5 & 5 & 7 & 10 & 14 & 20 & 28 & 40 \\
\hline \multirow[t]{2}{*}{ TMT } & $\bar{x}$ & 17 & 12 & 7 & 8 & -3 & 4 & 0 & 3 & 10 \\
\hline & S.E. & 8 & 6 & 5 & 9 & 5 & 3 & 3 & 9 & 9 \\
\hline \multirow[t]{2}{*}{ Saline } & $\bar{x}$ & 15 & 5 & 6 & 3 & -7 & 4 & 1 & -2 & 6 \\
\hline & S.E. & 8 & 4 & 8 & 2 & 4 & 6 & 5 & 4 & 4 \\
\hline
\end{tabular}




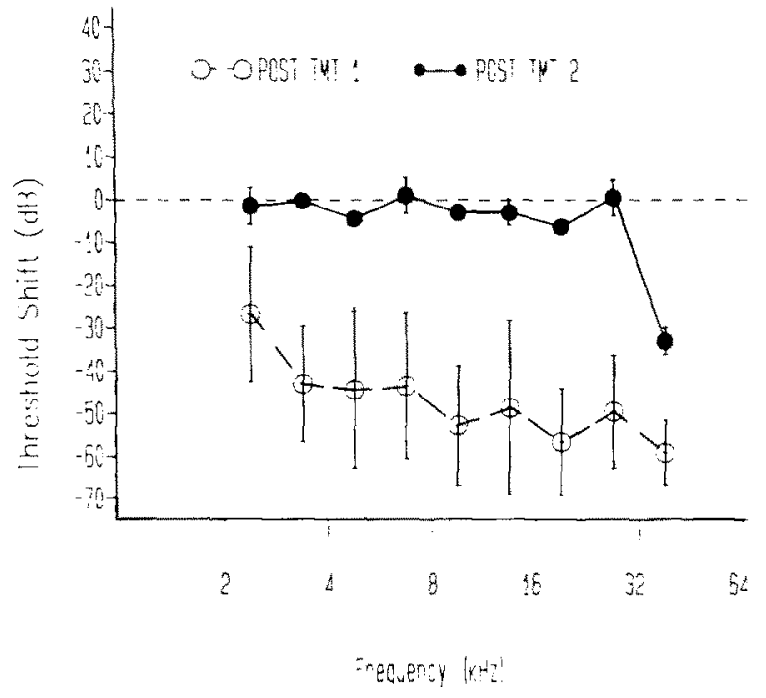

Fig. 1. Threshold shift (in dB) for TMT-exposed subjects in the 2 weeks immediately after exposure (post TMT 1) and $6-8$ weeks after exposure (post TMT 2) referenced to pre-exposure values for each subject.

subjects during the same interval. The reduction in baseline startle among TMT-treated subjects ranged between 0 and $-79 \%$ at the second postTMT administration time. Overall, a significant

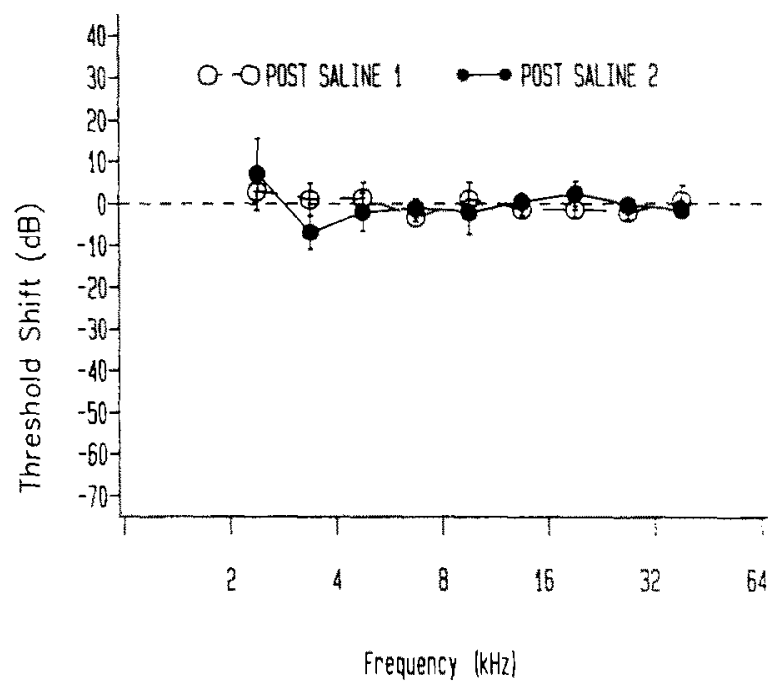

Fig. 2. Threshold shift (in $\mathrm{dB}$ ) for saline control subjects in the 2 weeks immediately following injection (post saline 1) and $6-8$ weeks after injection (post saline 2) referenced to pre-injection values for each subject. effect of TMT on baseline startle levels was observed across test periods $(F=15.95, P<0.01$ ). Shifts in baseline response of this magnitude may occur following toxicant exposure without any shift in auditory threshold (Fechter and Young, 1983). In assessing threshold shifts in individual subjects, there was no relationship found between the drop in baseline startle response level in TMT subjects and extent of the sensory threshold shift $(r=-0.32)$.

Generally the electrophysiologic data parallelled the behavioral results. A large difference was observed in the intensity of a pure tone needed to elicit the CAP at higher frequencies between groups (see Fig. 3). Higher detection thresholds in the TMT-treated subjects are especially apparent at higher tone frequencies. As in the behavioral data, however, evidence of impaired function is exhibited by TMT-treated subjects even in a middle-frequency range. An analysis of variance showed significant overall group differences ( $F=$ 17.31, $P<0.0001)$, frequency $(F=24.63, \quad P<$ 0.0001 ) and group by frequency interactions ( $F=$ 18.60, $P<0.0001$ ) with post-hoc analyses indicating significant impairment among TMT subjects at frequencies above $4 \mathrm{kHz}$. The $\mathrm{CM}$ showed similar decrements among TMT-treated subjects

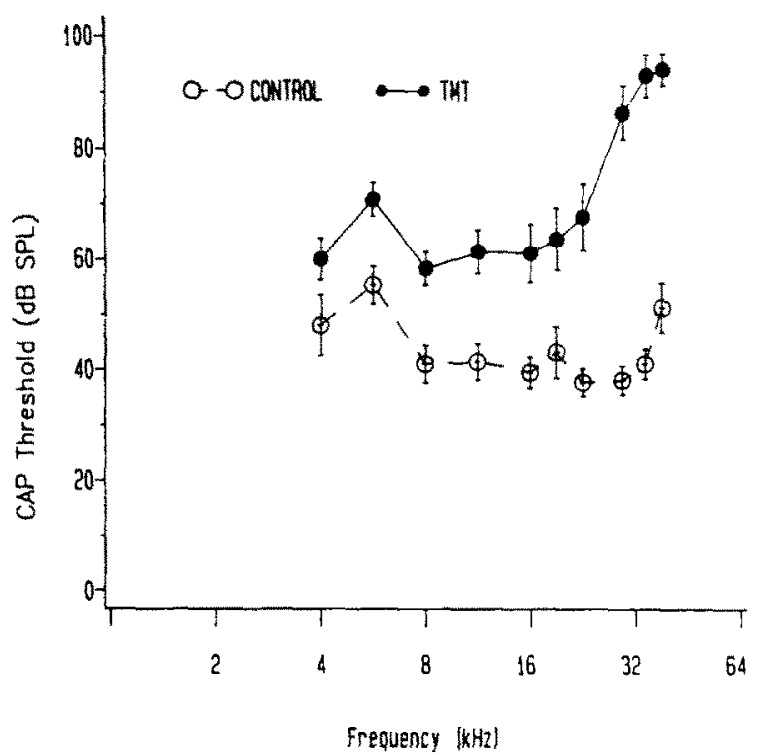

Fig. 3. Threshold for evoking a CAD measured at the round window in control and $1 \mathrm{M} /$-treated subjects. 


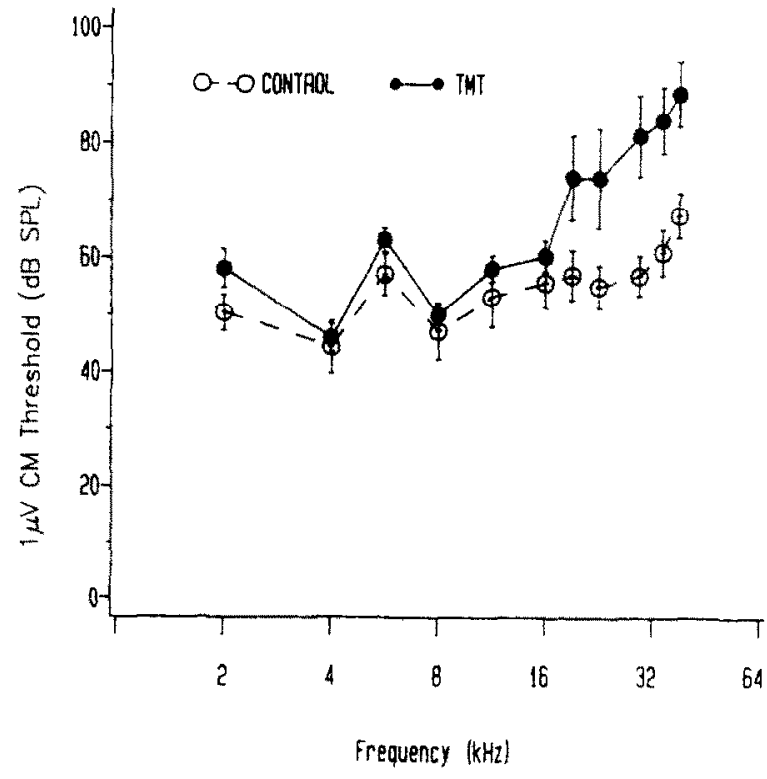

Fig. 4. Threshold for evoking a $1 \mu \mathrm{V}$ RMS $\mathrm{CM}$ response measured at the round window in control and TMT-treated subjects.

(see Fig. 4) although the differences between groups in this measure tended to be smaller than that seen for the CAP. Again, ANOVA showed significant between group differences ( $F=17.48$, $P<0.0001)$, frequency $(F=27.06, \quad P<0.0001)$ and group $\mathrm{X}$ frequency interactions $(F=5.22$, $P<0.0001$ ). Post-hoc analyses showed the TMTinduced impairment of the $\mathrm{CM}$ to be significant above $24 \mathrm{kHz}$.

\section{Discussion}

The results of the current investigation generally support the findings of Young and Fechter (1986) in showing a profound though partially reversible elevation in auditory thresholds among rats treated with TMT. Further, the current work provides direct evidence for a cochlear locus for TMT ototoxicity while not excluding possible additional damage in more central auditory pathways. The data showing a frequency relationship in hearing impairment and recovery certainly underscore the importance of the cochlear compartment in TMT ototoxicity. The current behavioral data do not provide quite as clear an indication of high frequency impairment following TMT as that previously observed by Young and Fechter (1986). This may reflect the more limited investigation of frequency effects in the carlier study. In the initial dose response study with TMT, thresholds assessed only at 10 and $40 \mathrm{kHz}$ were significantly affected at both frequencies by the highest TMT dose $(6 \mathrm{mg} / \mathrm{kg}$ ), but only at the higher frequency in the $4 \mathrm{mg} / \mathrm{kg}$ TMT group. However, there is a trend in the current data consistent with a preferential high frequency loss induced by TMT demonstrated behaviorally at the initial post-TMT test and clear evidence for a frequency relationship in terms of recovery of function. Moreover, the electrophysiological evidence also supports the notion of a high frequency loss in terms of cochlear output.

The locus of TMT-induced injury cannot be specifically determined from this study although the data suggest two types of damage. It is clear from the electrophysiological data that TMT disrupts cochlear function. The loss in sensitivity of CM only at high frequencies is consistent with the notion of damage to outer hair cells in a restricted area of the basal cochlea; that is, near the round window recording site. The broader and more profound disruption of the CAP may reflect injury at any one (or more) of several sites including the hair cells and their associated structures (e.g. stereocilia), the spiral ganglion cells or in support tissues necessary for normal metabolism and function of those cells. Presumably, based upon the behavioral data, some of this loss is recoverable. Whether the permanent very high frequency loss and the more transitory lower frequency loss reflect similar mechanisms of TMT ototoxicity and differences in dosage reaching the target tissue is uncertain.

Several markers of TMT toxicity have been identified. TMT is known to bind to mitochondria where it is a potent inhibitor of oxidative phosphorylation (Aldridge and Street, 1971). Ultrastructural studies have suggested edematous changes in neurons possibly as a consequence of disruption in ATP production. These may be reversible although more severe and presumably permanent neuronotoxic changes have also been observed (Chang et al., 1983; Chang, 1984). Potentially, the recovery of function could reflect recovery of mitochondrial function and restora- 
tion of normal cellular activity following metabolism or excretion of TMT. Such an explanation still requires an understanding of the differential vulnerability of structures encoding high- as opposed to low-frequency tones, but it is possible that differences in metabolic activity do, in fact. exist along the apical to basal axis of the cochlea (Thalmann et al., 1973). An additional requirement for further experimentation is to determine why TMT but not TET disrupts hearing: both of these compounds being potent inhibitors of oxidative phosphorylation. It is possible that TMT, but not TET is selectively taken up by the cochlea where it is capable of producing damage. If this is the case, then direct placement of TET into the cochlea should be as effective as systematic TMT administration in perturbing high-frequency hearing.

The most unusual feature of TMT-induced ototoxicity is the slow, partial recovery of behavioral function observed at mid-range to high frequencies: the time course of this recovery being more reminiscent of noise-induced hearing loss than of chemical exposure. This time course could. in fact, reflect the persistence of TMT or an active metabolite within the cochlea and, thereby, an acute response to the presence of this agent. Hasan et al. (1984) reported elevated tin levels in both blood and whole brain up to 20 days following exposure. However, such a notion still does not address the site or mechanism for TMT's action nor its frequency specificity.

\section{Acknowledgements}

Supported in part by NIH grant nos. ES 02852 and NS 11731. L.F. is supported by RCDA no. ES 00125. The authors express their gratitude to Blair Miller, A. Gail Lee, Michelle Griffiths and J. Nadine Brown for their expert technical assistance. to Susan Pierson, Jane Rogers and Janet Mello for their patient and efficient typing, and to Dr. P. Thorne for comments on an earlier version of this manuscript.

\section{References}

Aldridge, W. and Street. B. (1971): The relation between the specific binding of trimethyltin and triethyltin to mito- chondria and their function on various mitochondrial functions. Biochemistry 124, 221-234.

Chang. L. (1984): Hippocampal lesions induced by trimethyltin in the neonatal rat brain. Neurotoxicology 5, 205-216.

Chang, L., Tiemeyer, T., Wenger, G, and McMillan, D. (1983): Neuropathology of trimethyltin intoxication. III: Changes in the brain stem neurons. Environ. Res. 30. 399-411.

Evans, B., Yonovitz, A. and Fox, D. (1984): Ototoxic effects of triethyltin: Electrophysiological and histological correlates. Toxicologist 4, 121.

Falk, S., Klein, R., Haseman, J., Sanders, G. and Talley, F. (1974): Acute methyl mercury intoxication and ototoxicity in guinea pigs. Arch. Pathol. 97. 297-305.

Fechter, L.D. and Young, J.S. (1983): Discrimination of auditory from nonauditory toxicity by reflex modification audiometry: Effects of triethyltin. Toxicol. Appl. Pharmacol. 70.216222 .

Fleischman, R., Stadnicki, S., Ethiers, M. and Schaeppi, U. (1975): Ototoxicity of cis-dichlorodiamine Platinum (II) in the guinea pig. Toxicol. Appl. Pharmacol. 33, 320-332.

Hasan, Z.. Zimmer. L. and Woolley, D. (1984): Time course of the effects of trimethyltin on limbic evoked potentials and distribution of tin in blood and brain in the rat. Neurotoxicology $4,217-244$.

Hoffman. H. and Ison, J.R. (1980): Reflex modification in the domain of startle: 1. Some empirical findings and their implications for how the nervous system processes sensory input. Psychol. Rev. 87, $175 \cdot 189$.

Ison, J.R. and Hoffman, H. (1983): Reflex modification in the domain of startle: II. The anomalous history of a robust and ubiquitous phenomenon. Psychol. Bull. 94, 3-17.

Kelly, J. and Masterton, B. (1977): Auditory sensitivity of the albino rat. J. Comp. Physiol. Psychol. 91, 930-936.

Leake-Jones, P. and Rebscher, S. (1983): Cochlear pathology with chronically implanted scala tympani electrodes. Ann. N.Y. Acad. Sci. 405, 203-223.

Prosen. C.A., Peterson. M.R., Moody, D.B. and Stebbins, W.C. (1978): Auditory thresholds and kanamycin-induced hearing loss in the guinea pig assessed by a positive rein forcement procedure. J. Acoust. Soc. Am. 559-566.

Pryor, G.. Dickinson, J., Howd, R. and Rebert. C. (1983): Transient cognitive deficits and high frequency hearing loss in weanling rats exposed to toluene. Neurobehav. Toxicol. Teratol. 5. 53-57.

Rebert, C., Houghton, P.. Howd, R. and Pryor. G. (1982): Effects of Hexane on the brainstem auditory response and caudal nerve action potential. Neurobehav. Toxicol. Teratol. 4. 79-85.

Rebert. C., Sorenson. S., Howd, R. and Pryor, G. (1983): Toluene-induced hearing loss in rats evidenced by the brainstem auditory-evoked response. Neurobehav. Toxicol. Teratol. 5, 59-62.

Sheppard, L., Young, J.S., Zeger, S. and Fechter, L.D. Sensory threshold estimation from a continuously graded response in reflex inhibition studies. (under review).

Thalmann, R., Kusahair, N. and Miyoshi, T. (1973): Dysfunctions of energy releasing and consuming processes of the cochlea. Laryngoscope 83, 1690-1712. 
Wilkinson, R. (1984): Technoeconomic and environmental assessment of industrial organotin compounds. Neurotoxicology $5,141-158$.

Wu, M-F., Ison, J., Wecher, J. and Lapham, L. (1985): Cutaneous and auditory functioning in rats following methyl mercury poisoning. Toxicol. Appl. Pharmacol. 79. 377-388.

Yamamura, K., Kishi, R., Maehara, N., Sadamoto, T. and Uchino, E. (1984): An experimental study of the effects of lead acetate on hearing. Cochlear microphonics and action potential of the guinea pig. Toxicol. Lett. 21, 45-47.

Young, J.S. and Fechter, L.D. (1983): Reflex inhibition procedures for animal audiometry: A technique for assessing ototoxicity. J. Acoust. Soc. Am. 73, 1686-1693.

Young, J.S. and Fechter, L.D. (1986): Trimethyltin exposure produces an unusual form of toxic auditory damage in rats. Toxicol. Appl. Pharmacol. 82, 87-93. 